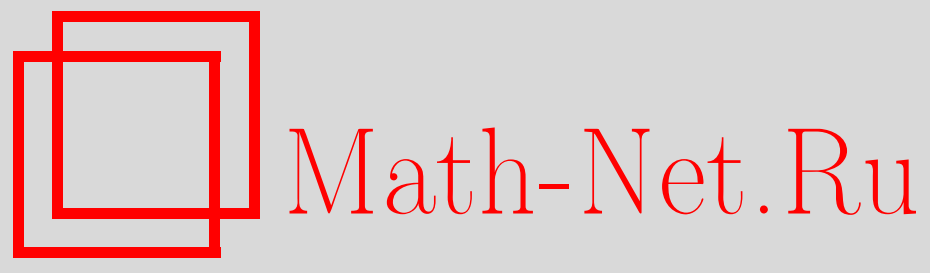

P. Eichelsbacher, Refined large deviations for von Mises statistics, Теория вероятн. и ее примен., 2004, том 49, выпуск 1, 197-204

DOI: https://doi.org/10.4213/tvp247

Использование Общероссийского математического портала Math-Net.Ru подразумевает, что вы прочитали и согласны с пользовательским соглашением

http://www.mathnet.ru/rus/agreement

Параметры загрузки:

IP : 54.209 .52 .79

26 апреля 2023 г., 13:16:12

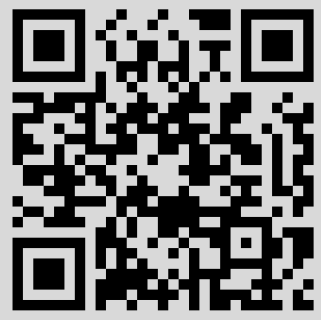


11. Сарманов О.В. Максимальный коэффициент корреляции (симметричный случай). - Докл. АН СССР, 1958, т. 120, № 4, с. 715-718.

12. Сарманов О.В. Максимальный коэффициент корреляции (несимметричный случай). - Докл. АН СССР, 1958, т. 121, № 1, с. 52-55.

13. Witsenhausen H.S. On sequences of pairs of dependent random variables. - SIAM J. Appl. Math., 1975, v. 28, p. 100-113.

Поступила в редакцию

1.IV.2003

(C) $2004 \mathrm{r}$.

EICHELSBACHER P.*

\section{REFINED LARGE DEVIATIONS FOR VON MISES STATISTICS}

Приводятся достаточные условия выполнения принципа больших уклонений для вещественнозначной статистики Мизеса, улучшающие предыдущие результаты. В качестве следствия получаются достаточные условия выполнения принципа больших уклонений для $U$-статистики со значениями в банаховом пространстве. Эти условия также улучшают полученные ранее результаты.

Доказательства основаны на результатах Арконеса о больших уклонениях для случайных процессов и спектральном разложении функции ядра для статистики Мизеса и $U$-статистики соответственно.

Ключевые слова и фразы: большие уклонения, статистика Мизеса, случайные процессы.

1. Introduction. Let $(B,\|\cdot\|)$ be a real separable Banach space with a norm $\|\cdot\|$ and let $\left\{X_{i}\right\}_{i \in \mathbf{N}}$ be a sequence of independent, identically distributed random variables taking values in a measurable space $(S, \mathscr{S})$. For simplicity we assume that they are defined on the product space $(\Omega, \mathscr{A}, \mathbf{P})=\left(S^{\mathbf{N}}, \mathscr{S}^{\otimes \mathbf{N}}, \mu^{\otimes \mathbf{N}}\right)$, where $\mu$ is the distribution of $X_{1}$. Denote by $\mathscr{M}_{1}(S)$ the space of all probability measures on $(S, \mathscr{S})$. Consider for $n \geqslant m$, $n, m \in \mathbf{N}$,

$$
U_{n}(h):=U_{n}^{m}(h, \mu):=\frac{1}{\left(\begin{array}{c}
n \\
m
\end{array}\right)} \sum_{1 \leqslant i_{1}<\cdots<i_{m} \leqslant n} h\left(X_{i_{1}}, \ldots, X_{i_{m}}\right),
$$

where $h: S^{m} \rightarrow B$ is a Bochner integrable symmetric function. $U_{n}(h)$ is called a $B$-valued $U$-statistic with kernel function $h$ and degree $m$. Moreover we consider the corresponding von Mises statistic

$$
V_{n}(h):=V_{n}^{m}(h, \mu):=\frac{1}{n^{m}} \sum_{i_{1}, \ldots, i_{m}=1}^{n} h\left(X_{i_{1}}, \ldots, X_{i_{m}}\right) .
$$

In this paper we analyze the large deviations principle for these statistics.

Let us recall the definition of the large deviations principle (LDP). A sequence of probability measures $\left\{\mu_{n}\right\}_{n \in \mathrm{N}}$ on a topological space $\mathscr{X}$ equipped with $\sigma$-field $\mathscr{B}$ is said to satisfy the LDP with speed $a_{n} \downarrow 0$ and good rate function $I: \mathscr{X} \rightarrow[0, \infty]$ if the level sets $\{x: I(x) \leqslant \alpha\}$ are compact for all $\alpha<\infty$ and for all $\Gamma \in \mathscr{B}$ the lower bound

$$
\liminf _{n \rightarrow \infty} a_{n} \ln \mu_{n}(\Gamma) \geqslant-\inf _{x \in \operatorname{int}(\Gamma)} I(x)
$$

and the upper bound

$$
\limsup _{n \rightarrow \infty} a_{n} \ln \mu_{n}(\Gamma) \leqslant-\inf _{x \in \operatorname{cl}(\Gamma)} I(x)
$$

* Fakultät für Mathematik, Ruhr-Universität Bochum, NA 3/68, D-44780 Bochum, Germany; e-mail: peter.eichelsbacher@ruhr-uni-bochum.de 
hold, where int $(\Gamma)$ and $\operatorname{cl}(\Gamma)$ denote the interior and closure of $\Gamma$, respectively. We say that a sequence of random variables satisfies the LDP when the sequence of measures induced by these variables satisfies the LDP.

The LDP of $\left\{U_{n}(h)\right\}_{n \in \mathbf{N}}$ have been studied already. In [5] the real-valued case $B=\mathbf{R}^{d}$ was considered: If $h$ satisfies the strong Cramér condition, that is,

$$
\int_{S^{m}} \exp (\theta\|h\|) d \mu^{\otimes m}<\infty \quad \text { for all } \theta>0,
$$

then the sequence $\left\{U_{n}(h)\right\}_{n \in \mathbb{N}}$ satisfies the LDP with rate function

$$
I(x)=\inf \left\{H(\varrho \mid \mu): \varrho \in K_{\infty}, F(\varrho)=x\right\}, \quad x \in \mathbf{R}^{d} .
$$

Here $H(\varrho \mid \mu)$ is the relative entropy of $\varrho \in \mathscr{M}_{1}(S)$ with respect to $\mu \in \mathscr{M}_{1}(S)$ defined by

$$
H(\varrho \mid \mu)= \begin{cases}\int_{S} f \ln f d \mu, & \text { if } \varrho \ll \mu \text { and } f=\frac{d \varrho}{d \mu}, \\ \infty & \text { otherwise, }\end{cases}
$$

$K_{\infty}=\bigcup_{L \geqslant 0} K_{L}$ with $K_{L}=\left\{\varrho \in \mathscr{M}_{1}(S): H(\varrho \mid \mu) \leqslant L\right\}$ and $F: \mathscr{M}_{1}(S) \cap K_{\infty} \rightarrow \mathbf{R}^{d}$ is defined by $F(\varrho)=\int_{S^{m}} h d \varrho^{\otimes m}$ (note that the map is well defined on $K_{\infty}$ ). If in addition $h$ satisfies the condition that there exists at least one $\alpha_{h}>0$ such that

$$
\int_{S^{m}} \exp \left(\alpha_{h}\left\|h \circ \pi_{\tau}\right\|\right) d \mu^{\otimes m}<\infty
$$

for every map $\tau:\{1, \ldots, m\} \rightarrow\{1, \ldots, m\}$, where $\pi_{\tau}: S^{m} \rightarrow S^{m}$ is defined by $\pi_{\tau}(s)=$ $\left(s_{\tau(1)}, \ldots, s_{\tau(m)}\right)$ for every $s=\left(s_{1}, \ldots, s_{m}\right) \in S^{m}$, then $\left\{V_{n}(h)\right\}_{n \in \mathbf{N}}$ satisfies the LDP with the same rate function $I$. This result is given in [6].

In the case of an arbitrary real separable Banach space valued kernel $h$, Eichelsbacher and Schmock [6] proved the LDP for $U$-statistics if $h$ satisfies the strong Cramér condition, and for von Mises statistics if in addition $h$ satisfies condition (1.1). As one can see from the definition of the rate function $I$, the proofs in the papers just mentioned apply a generalized contraction principle, see Theorem 4.2.23 in [3], and use a LDP for products of empirical measures in a topology which make integration with respect to certain unbounded functions a continuous operation.

This approach gives rise to the question if $F(\cdot)$ is continuous on the level sets $K_{L}$, $L \geqslant 0$, of the relative entropy (continuity on the level sets is the minimal condition needed to apply the generalized contraction principle). But we obtain from Theorem 3 in [9] that continuity of $F(\cdot)$ on the level sets of $H(\cdot \mid \mu)$ is equivalent to the strong Cramér condition holding for $h$. This result shows that in case a weaker assumption than the strong Cramér condition is assumed the LDP for $U$-statistics cannot be deduced by known contraction techniques from Sanov's theorem, even in the real-valued case. This seems to be surprising.

In the case $m=1$ and $h(x)=x$ it is well known that the classical Cramér theorem states that the weak Cramér condition $\int_{S} \exp (\delta\|x\|) d \mu<\infty$ for some $\delta>0$ is a necessary and sufficient condition to have a LDP with a good rate function.

Therefore natural questions are: Do we get a LDP for von Mises statistics and $U$ statistics, respectively, under weaker assumptions? Which method could replace the contraction technique?

In this paper we improve previous LDP results for real-valued von Mises statistics. Surprisingly the result looks like an exercise. Applying a spectral decomposition of the kernel function $h$ as well as a new LDP-development for stochastic processes due to Arcones [1], we give new sufficient conditions for the LDP. Moreover we will weaken the assumptions for the LDP of $U$-statistics. If the diagonal terms fulfill the weak Cramér condition, we can give improved sufficient conditions for the LDP of $U$-statistics. This is presented in Section 2.

In Section 3 we improve the LDP result for a Banach space valued kernel $h$ given in [6]. For the proof we apply results from [1] as well as an inequality due to Hoeffding (see [7]). 
2. The real valued case. Along the lines of [2] and [1] we obtain new sufficient conditions for the LDP of a real valued von Mises statistic in the case $m=2$. We state this result and afterwards give some comments for the formulation for an arbitrary $m \geqslant 2$. The result relies on a LDP of sums of Banach space valued i.i.d. random variables. Let us shortly summarize the state of the art. If $(B,\|\cdot\|)$ is a real separable Banach space and if $\left\{X_{i}\right\}$ is a sequence of $B$-valued i.i.d. random variables, Sethuraman [11] and Donsker and Varadhan [4] showed that if $\operatorname{E} \exp \left(\lambda\left\|X_{1}\right\|\right)<\infty$ for each $\lambda>0$, then the LDP holds for $\left\{(1 / n) \sum_{i=1}^{n} X_{i}\right\}$ with speed $1 / n$ and with rate function

$$
I(x)=\sup \left\{f(x)-\ln \mathrm{E} \exp \left(f\left(X_{1}\right)\right): f \in B^{*}\right\},
$$

where $B^{*}$ is the dual of $B$. Arcones [1, Corollary 2.8] proved that the LDP holds for $\left\{(1 / n) \sum_{i=1}^{n} X_{i}\right\}$ with speed $1 / n$ and a good rate function if and only if there exists a $\lambda>0$ such that $\mathbf{E} \exp (\lambda\|X\|)<\infty$, and for each $\lambda>0$ there exists an $\eta>0$ such that $\mathbf{E} \exp \left(\lambda Y^{\eta}\right)<\infty$, where

$$
Y^{\eta}:=\sup \left\{\left|f_{1}\left(X_{1}\right)-f_{2}\left(X_{1}\right)\right|: f_{1}, f_{2} \in B_{1}^{*}, \mathbf{E}\left|f_{1}\left(X_{1}\right)-f_{2}\left(X_{1}\right)\right| \leqslant \eta\right\}
$$

where $B_{1}^{*}$ is a unit ball of $B^{*}$. In [1, Theorem 2.7] we find some formula for the rate function in this case, but it seems not to be obvious that the formula is the same as in the case where the $X_{i}$ satisfies the strong Cramér condition. The sequence $\left\{(1 / n) \sum_{i=1}^{n} X_{i}\right\}$ satisfies the LDP in $B$ with speed $1 / n$ if and only if

$$
\left\{\frac{1}{n} \sum_{i=1}^{n} f\left(X_{i}\right): f \in B_{1}^{*}\right\}
$$

satisfies the LDP in $l_{\infty}\left(B_{1}^{*}\right)$ with speed $1 / n$. Here $l_{\infty}\left(B_{1}^{*}\right)$ denotes the set of bounded functions in $B_{1}^{*}$ with the norm $\|x\|:=\sup _{t \in B_{1}^{*}}|x(t)|, x \in l_{\infty}\left(B_{1}^{*}\right)$. Arcones [1] gave a formula for the rate function on $l_{\infty}\left(B_{1}^{*}\right)$ : for $x \in l_{\infty}\left(B_{1}^{*}\right)$ he obtained

$$
I(x)=\sup \left\{I_{f_{1}, \ldots, f_{m}}\left(x\left(f_{1}\right), \ldots, x\left(f_{m}\right)\right): f_{1}, \ldots, f_{m} \in B_{1}^{*}, m \geqslant 1\right\},
$$

where

$$
I_{f_{1}, \ldots, f_{m}}\left(u_{1}, \ldots, u_{m}\right)=\sup \left\{\sum_{j=1}^{m} \lambda_{j} u_{j}-\ln \left(\mathbf{E} \exp \left(\sum_{j=1}^{m} \lambda_{j} f_{j}\left(X_{1}\right)\right)\right): \lambda_{1}, \ldots, \lambda_{m} \in \mathbf{R}\right\} .
$$

For notational reasons let us consider only the case $m=2$ in detail. Using the notation $\pi_{1} h(x):=\mathbf{E}_{Y} h(x, Y)-\mathbf{E} h(X, Y)$ and $\pi_{2} h(x, y):=h(x, y)-\mathbf{E}_{Y} h(x, Y)-\mathbf{E}_{X} h(X, y)+$ $\mathbf{E} h(X, Y)$, where $\mathbf{E}_{X}$ (respectively, $\mathbf{E}_{Y}$ ) indicates expectation with respect to $X$ (respectively, $Y$ ) only, we recall Hoeffding's decomposition

$$
\sum_{i, j=1}^{n} h\left(X_{i}, X_{j}\right)=n^{2} \mathbf{E} h(X, Y)+2 n \sum_{i=1}^{n} \pi_{1} h\left(X_{i}\right)+\sum_{i, j=1}^{n} \pi_{2} h\left(X_{i}, X_{j}\right)
$$

as well as

$$
\sum_{1 \leqslant i<j \leqslant n} h\left(X_{i}, X_{j}\right)=\sum_{1 \leqslant i<j \leqslant n} \pi_{2} h\left(X_{i}, X_{j}\right)+(n-1) \sum_{i=1}^{n} \pi_{1} h\left(X_{i}\right)+\left(\begin{array}{l}
n \\
2
\end{array}\right) \mathbf{E} h(X, Y) .
$$

The identities hold a.s. for $\mu^{\otimes 2}$. The kernel $\pi_{2} h$ is canonical (degenerate) for the law of $X$ meaning that $\mathbf{E}_{X} \pi_{2} h(X, Y)=\mathbf{E}_{Y} \pi_{2} h(X, Y)=0$ a.s. Note that $\pi_{1} h(X)$ is centered. If $\pi_{2} h \in L_{2}\left(\mu^{\otimes 2}\right)$, then there exist an orthonormal sequence $\left\{\psi_{r}\right\}_{r=1}^{\infty}$ of centered square-integrable functions and a sequence of real constants $\left\{c_{r}\right\}_{r=1}^{\infty}$ such that $\pi_{2} h(x, y)=\sum_{r=1}^{\infty} c_{r} \psi_{r}(x) \psi_{r}(y)$ with convergence in the sense of $L_{2}\left(\mu^{\otimes 2}\right)$. Note that the sequence of eigenvalues $\left\{c_{r}\right\}_{r=1}^{\infty}$ is square-summable (see [8, Section 3.2.2, Theorem 1]). We take $c_{0}:=1$ and $\psi_{0}:=2 \pi_{1} h-\mathbf{E} h(X, Y)$. Then

$$
V_{n}(h)=\frac{1}{n} \sum_{i=1}^{n} \psi_{0}\left(X_{i}\right)+\sum_{r=1}^{\infty} c_{r}\left(\frac{1}{n} \sum_{i=1}^{n} \psi_{r}\left(X_{i}\right)\right)^{2}
$$


and

$$
U_{n}(h)=\frac{1}{n} \sum_{i=1}^{n} \psi_{0}\left(X_{i}\right)+\sum_{r=1}^{\infty} c_{r}\left(\frac{1}{n} \sum_{i=1}^{n} \psi_{r}\left(X_{i}\right)\right)^{2}-\sum_{r=1}^{\infty} c_{r} \frac{1}{n^{2}} \sum_{i=1}^{n} \psi_{r}\left(X_{i}\right)^{2} .
$$

In what follows we will assume that $h$ has a representation such that $V_{n}(h)$ and $U_{n}(h)$, respectively, can be represented as in (2.2) and (2.3), respectively. Denote

$$
l_{2}=\left\{x=\left(x_{p}\right)_{p=0}^{\infty}, x_{p} \in \mathbf{R}, \sum_{p=0}^{\infty} x_{p}^{2}<\infty\right\}
$$

and let $l_{2}^{*}$ be the dual space, which is $l_{2}$. Let us denote by $\|\cdot\|_{2}$ the norm in $l_{2}$. Now we consider the following conditions.

$\mathrm{C}$ on $\mathrm{d}$ it i o n 1 . There exists a $\lambda>0$ such that

$$
\mathbf{E} \exp \left\{\lambda\left(\sum_{r=0}^{\infty}\left|c_{r}\right|\left(\psi_{r}\left(X_{1}\right)\right)^{2}\right)^{1 / 2}\right\}<\infty
$$

C o n d it i o n 2. For each $\lambda>0$ there exists an $\eta>0$ such that $\mathbf{E} \exp \left(\lambda F^{\eta}\right)<\infty$ with

$$
F^{\eta}:=\sup _{. f_{1}, f_{2} \in l_{2}:\left\|f_{i}\right\|_{2} \leqslant 1, d\left(f_{1}, f_{2}\right) \leqslant \eta}\left|\left(f_{1}-f_{2}\right)\left(\left(\left|c_{r}\right|^{1 / 2} \psi_{r}\left(X_{i}\right)\right)_{r=0}^{\infty}\right)\right|
$$

and $d\left(f_{1}, f_{2}\right):=\left.\mathbf{E}\left|\sum_{r=0}^{\infty}\left(f_{1}-f_{2}\right)\left(e_{r}\right)\right| c_{r}\right|^{1 / 2} \psi_{r}\left(X_{i}\right) \mid$.

Here $e_{r}=(0, \ldots, 0,1,0, \ldots) \in l_{2}$. Now we obtain the following results.

Theorem 2.1 (LDP for von Mises statistics). The sequence $\left\{V_{n}(h)\right\}_{n \in \mathbf{N}}$ satisfies a $L D P$ with a good rate function $I(\cdot)$ if $h: S^{2} \rightarrow \mathbf{R}$ satisfies Conditions 1 and 2 . The rate function has the form

$$
I(x)=\inf \left\{J(y): y \in l_{2}, y_{0}+\sum_{r=1}^{\infty} \operatorname{sign}\left(c_{r}\right) y_{r}^{2}=x\right\},
$$

where for every $y \in l_{2}, J(y)$ is the rate function of the sequence

$$
\left(\left|c_{r}\right|^{1 / 2} \frac{1}{n} \sum_{i=1}^{n} \psi_{r}\left(X_{i}\right)\right)_{r=0}^{\infty} \in l_{2} .
$$

$\mathrm{R}$ e $\mathrm{m}$ a r k 2.1. If the strong Cramér condition is fulfilled for $h$, that is, $\mathrm{E} \exp \left\{\lambda\left(\sum_{r=0}^{\infty}\left|c_{r}\right| \psi_{r}^{2}\left(X_{i}\right)\right)^{1 / 2}\right\}<\infty$ for every $\lambda>0$, then one obtains the well-known representation

$$
J(y):=\sup _{\xi \in l_{2}}\left\{\sum_{r=0}^{\infty} \xi_{r} y_{r}-\ln \mathbf{E} \exp \left\{\sum_{r=0}^{\infty} \xi_{r} c_{r} \psi_{r}\left(X_{1}\right)\right\}\right\}
$$

see the discussion in [2].

Theorem 2.2 (LDP for $U$-statistics). If $h$ satisfies Condition 1 , Condition 2 and (1.1), then the sequence $\left\{U_{n}(h)\right\}_{n \in \mathbb{N}}$ satisfies the LDP with good rate function $I(\cdot)$ given by (2.4).

Our theorems are improvements of all LDP results for $U$-statistics and von Mises statistics, that is theorem 1 in [2], Theorem 2 and Corollary 3 in [5] and Theorems (1.7), (1.10) and (1.13) in [6].

$\mathrm{R}$ e $\mathrm{m}$ a $\mathrm{r} \mathrm{k} 2.2$. If $h$ satisfies Condition 1, its diagonal does not satisfy the weak Cramér condition (1.1) in any case. We will give a counterexample. Let $Y_{k}$ be i.i.d. random variables with $\mathscr{N}\left(0, k^{2}\right)$-distribution. Independently of this sequence let $Z$ be integer-valued with $\mathbf{P}\{Z=k\}=c e^{-k^{2}}$, where $c$ is chosen such that the distribution of $Z$ will become a probability distribution. Let $X$ be the $l_{2}$-valued random variable with components $X_{k}=Y_{k}$, if $Z=k$, and 0 otherwise. Let $c_{r}=1$ and $\psi_{r}(X)=X_{r}$, thus we 
consider the case $S=l_{2}$. Now we obtain that $\sum_{r=0}^{\infty} \psi_{r}(X) \psi_{r}(X)=Y_{Z}^{2}$, and therefore we get

$$
\mathbf{E} \exp \left\{\lambda\left(\sum_{r=0}^{\infty} \psi_{r}(X) \psi_{r}(X)\right)^{1 / 2}\right\}=\sum_{k=0}^{\infty} \mathbf{E}\left[e^{\lambda Y_{k}}, Z=k\right] .
$$

After a little calculation we obtain $\sum_{k=0}^{\infty} \mathbf{E}\left[e^{\lambda Y_{k}}, Z=k\right]=c \sum_{k=0}^{\infty} e^{\left(\lambda^{2} k^{2}-k^{2}\right) / 2} \leqslant$ $c \sum_{k=0}^{\infty} e^{-k^{2} / 2}<\infty$ for $\lambda \leqslant 1$. But for any given $\lambda>0$ we can take $m$ such that $\lambda>\frac{1}{2} m^{2}$ and find

$$
\mathbf{E} e^{\lambda Y_{Z}^{2}} \geqslant \mathbf{E}\left[e^{\lambda Y_{m}^{2}}, Z=m\right]=\infty .
$$

Thus we have found an example, where Condition 1 is fulfilled, but not condition (1.1).

$\mathrm{Pr}$ o of of $\mathrm{Th}$ e o r e $\mathrm{m}$ 2.1. By the assumptions of the theorem we obtain that

$$
X(n):=\left(\left|c_{r}\right|^{1 / 2} \frac{1}{n} \sum_{i=1}^{n} \psi_{r}\left(X_{i}\right)\right)_{r=0}^{\infty} \in l_{2} .
$$

Since $l_{2}$ is a real separable Banach space, we obtain by Arcones' [1] result that the sequence $\{X(n)\}_{n \in \mathbf{N}}$ satisfies a LDP if and only if Conditions 1 and 2 are fulfilled. Given the continuous function $F_{h}(y): l_{2} \rightarrow \mathbf{R}$, defined by $F_{h}(y):=y_{0}+\sum_{r=1}^{\infty} \operatorname{sign}\left(c_{r}\right) y_{r}^{2}$, we get the representation

$$
V_{n}(h)=F_{h}(X(n)) \text {. }
$$

Using the contraction principle [3, Theorem 4.2.1], the theorem follows immediately.

$\mathrm{P} \mathrm{r}$ o of of $\mathrm{T} \mathrm{h}$ e or e m 2.2. The only thing we have to prove is that condition (1.1) for $h$ implies that the sequences $\left\{U_{n}(h)\right\}_{n \in \mathbf{N}}$ and $\left\{V_{n}(h)\right\}_{n \in \mathbf{N}}$ have identical large deviations behavior, that is, for every $\delta>0$ we obtain

$$
\limsup _{n \rightarrow \infty} \frac{1}{n} \ln \mathbf{P}\left\{\left\|U_{n}(h)-V_{n}(h)\right\| \geqslant \delta\right\}=-\infty .
$$

Notice that condition (1.1) implies that $\int_{S^{2}} \exp (\lambda\|h(x, x)\|) d \mu<\infty$ for some $\lambda>0$. This condition suffices to get (2.5). This is proved more generally for kernel functions with $m \in \mathbf{N}$ variables in [6, proof of Theorem (1.10)], applying Baranyai's result on the factorization of complete uniform hypergraphs.

$\mathrm{E} \mathrm{x} \mathrm{a} \mathrm{m} \mathrm{p} 1 \mathrm{e} 2.1$. Let us consider the sample variance $U_{n}^{\mathrm{var}}$, which is a $U$-statistic of degree 2 with kernel function $h(x, y)=\frac{1}{2}(x-y)^{2}$. A simple calculation shows that $\pi_{1} h(x)=\frac{1}{2}\left((x-\mathbf{E} X)^{2}-\mathbf{D} X\right)$, where $\mathbf{D} X$ denotes the variance of $X$ under $\mu$. Moreover $\pi_{2} h(x, y)=-(x-\mathbf{E} X)(y-\mathbf{E} X)$. Thus $\psi_{0}(x)=\left((x-\mathbf{E} X)^{2}-\mathbf{D} X\right)-\mathbf{E} h(X, Y), c_{1}=-1$ and $\psi_{1}(x)=x-\mathbf{E} X_{1} ; \psi_{i}=0$ for $i \geqslant 2$. Let us assume that $X_{i}$ is $\mathscr{N}(0,1)$ distributed. Then the strong Cramér condition does not hold for the variance-kernel. But it is easily seen that Condition 1 is fulfilled. Remark that Condition 2 is fulfilled since every $f \in l_{2}$ is a linear and continuous function and $f(x) \leqslant\|f(x)\| \leqslant \beta\|x\|$ for some $\beta>0$. Using the decomposition of $h$ we obtain Condition 2 .

The theorems in this section can be formulated and proved for von Mises statistics and $U$-statistics, respectively, of arbitrary degree $m \geqslant 2$. Therefore we need to describe the spectral decomposition of a kernel function $h: S^{m} \rightarrow \mathbf{R}$ and its Hoeffding decomposition. Denote $\theta:=\mathbf{E} h\left(X_{1}, \ldots, X_{m}\right)$ and define the canonical functions $h_{c}: S^{c} \rightarrow \mathbf{R}$ by the formula

$$
h_{c}\left(x_{1}, \ldots, x_{c}\right):=\int_{S^{c}} h\left(y_{1}, \ldots, y_{m}\right) \prod_{s=1}^{c}\left(\delta_{x_{s}}-\mu\left(d y_{s}\right)\right) \prod_{s=c+1}^{m} \mu\left(d y_{s}\right) .
$$

According to the canonical Hoeffding decomposition we have the representation

$$
V_{n}(h)=\theta+\sum_{c=1}^{m}\left(\begin{array}{c}
m \\
c
\end{array}\right) V_{n}\left(h_{c}\right)
$$

We only state the spectral decomposition in the case $m=3$. It is straightforward to adapt the technique for an arbitrary degree $m$, but we omit this. We already have 
seen that we can find a sequence $\left\{\psi_{r_{1}}^{(2)}\right\}_{r_{1}=0}^{\infty}$ of centered square integrable functions and a sequence of constants $c_{r_{1}}^{(2)}$ such that $h_{2}(x, y)=\sum_{r_{1}=1}^{\infty} c_{r_{1}}^{(2)} \psi_{r_{1}}^{(2)}(x) \psi_{r_{1}}^{(2)}(y)$. Now we proceed iteratively to write for $h_{3}\left(x_{1}, x_{2}, x_{3}\right)$ an expansion in $L_{2}$ like $h_{3}\left(x_{1}, x_{2}, x_{3}\right)=$ $\sum_{r_{1}, r_{2}=1}^{\infty} c_{r_{1}}^{(3)} c_{r_{1}, r_{2}}^{(3)} \psi_{r_{1}}^{(3)}\left(x_{1}\right) \psi_{r_{1}, r_{2}}^{(3)}\left(x_{2}\right) \psi_{r_{1}, r_{2}}^{(3)}\left(x_{3}\right)$, where the functions are centered square integrable. The third term in the Hoeffding decomposition can be represented as

$$
\sum_{r_{1}, r_{2}=1}^{\infty} \operatorname{sign}\left(c_{r_{1}, r_{2}}^{(3)}\right) \Psi_{n, r_{1}}^{(3)}\left(\Psi_{n, r_{1}, r_{2}}^{(3)}\right)^{2}
$$

with $\Psi_{n, r_{1}}^{(3)}=c_{r_{1}}^{(3)} n^{-1} \sum_{j=1}^{n} \psi_{r_{1}}^{(3)}\left(X_{j}\right)$ and $\Psi_{n, r_{1}, r_{2}}^{(3)}=\left|c_{r_{1}, r_{2}}^{(3)}\right|^{1 / 2} n^{-1} \sum_{j=1}^{n} \psi_{r_{1}, r_{2}}^{(3)}\left(X_{j}\right)$. Now we can put these sample-means in a vector

$$
Y(n)=\left(c_{r_{1}}^{(3)} \frac{1}{n} \sum_{j=1}^{n} \psi_{r_{1}}^{(3)}\left(X_{j}\right),\left|c_{r_{1}, r_{2}}^{(3)}\right|^{1 / 2} \frac{1}{n} \sum_{j=1}^{n} \psi_{r_{1}, r_{2}}^{(3)}\left(X_{j}\right)\right)
$$

and proceed as in the case $m=2$ representing any event like $\left\{V_{n}(h) \in A\right\}$ in the form $\left\{Y(n) \in F_{h}(A)\right\}$ with an appropriate function $F_{h}$.

Since (2.5) is true for any degree $m \in \mathbf{N}$, we obtain Theorems 2.1 and 2.2 for an arbitrary degree $m \in \mathbf{N}$ using the spectral decomposition just described.

3. The Banach space case. Let $(B,\|\cdot\|)$ be an arbitrary real separable Banach space and let $B^{*}$ be the dual space. For $B$-valued $U$-statistics we obtain the following result.

Theorem 3.1 (LDP for Banach space valued statistics). Given a measurable, symmetric kernel function $h: S^{2} \rightarrow B$, assume that for any $f \in B^{*}$ the real valued function $f \circ h$ satisfies Conditions 1 and 2 and condition (1.1) (considering a decomposition for $f \circ h$ as in Section 2 for every $f \in B^{*}$ ). Moreover, assume that for any $\lambda>0$ there exists an $\eta>0$ such that

$$
\mathbf{E} \exp \left(\lambda Y^{\eta}\right)<\infty,
$$

where we define

$$
\begin{gathered}
Y^{\eta}:=\sup \left\{\left|f_{1}(h(X, Y))-f_{2}(h(X, Y))\right|: f_{1}, f_{2} \in B^{*},\left\|f_{i}\right\| \leqslant 1,\right. \\
\left.\mathbf{E}\left|f_{1}(h(X, Y))-f_{2}(h(X, Y))\right| \leqslant \eta\right\} .
\end{gathered}
$$

Then the sequence $\left\{U_{n}(h)\right\}_{n \in \mathbf{N}}$ satisfies the LDP with a good rate function, that is, the sequence $\left\{U_{n}(f \circ h), f \in T\right\}_{n \in \mathbb{N}}$ satisfies the $L D P$ in $l_{\infty}(T)$ with a good rate function, with $T:=\left\{f \in B^{*}:\|f\| \leqslant 1\right\}$.

For the proof of this theorem we will use the following result of Arcones [1, Theorem 2.1]: Let $\left\{X_{n}(t): t \in T\right\}$ be a sequence of stochastic processes, where $T$ is an index set. Let $\left\{\varepsilon_{n}\right\}$ be a sequence of positive numbers that converges to zero. Let $I: l_{\infty}(T) \rightarrow[0, \infty]$ and let $I_{t_{1}, \ldots, t_{k}}: \mathbf{R}^{k} \rightarrow[0, \infty]$ be a function for each $t_{1}, \ldots, t_{k} \in T$. Let $d$ be a pseudometric in $T$. Consider the following conditions:

(1) $(T, d)$ is totally bounded;

(2) for each $t_{1}, \ldots, t_{k} \in T$, the vector $\left(X_{n}\left(t_{1}\right), \ldots, X_{n}\left(t_{k}\right)\right)$ satisfies the LDP with speed $\varepsilon_{n}$ and good rate function $I_{t_{1}, \ldots, t_{k}}$;

(3) for each $\tau>0$

$$
\lim _{\eta \rightarrow 0} \limsup _{n \rightarrow \infty} \varepsilon_{n} \ln \mathbf{P}^{*}\left\{\sup _{d(s, t) \leqslant \eta}\left|X_{n}(t)-X_{n}(s)\right| \geqslant \tau\right\}=-\infty .
$$

Then for each $0<\alpha<\infty$ the set $\left\{z \in l_{\infty}(T): I(z) \leqslant \alpha\right\}$ is a compact set in $l_{\infty}(T)$, where

$$
I(z)=\sup \left\{I_{t_{1}, \ldots, t_{k}}\left(z\left(t_{1}\right), \ldots, z\left(t_{k}\right)\right): t_{1}, \ldots, t_{k} \in T, k \geqslant 1\right\} .
$$

Moreover one gets the upper and lower bounds in the LDP with respect to outer and inner probabilities (because of the lack of measurability): for each $A \subset l_{\infty}(T)$,

$$
-\inf _{z \in \operatorname{int}(A)} I(z) \leqslant \liminf _{n \rightarrow \infty} \varepsilon_{n} \ln \mathbf{P}_{*}\left\{X_{n} \in A\right\} \leqslant \limsup _{n \rightarrow \infty} \varepsilon_{n} \ln \mathbf{P}^{*}\left\{X_{n} \in A\right\} \leqslant-\inf _{z \in:(A)} I(z) .
$$


P r o of of $\mathrm{Th}$ e or e $\mathrm{m}$ 3.1. We will check conditions (1)-(3) of the general LDP approach for stochastic processes due to Arcones [1]: The pseudo-metric on $T=$ $\left\{f \in B^{*}:\|f\| \leqslant 1\right\}$ is chosen to be the $L_{1}$-pseudo-metric $d\left(f_{1}, f_{2}\right):=\mathbf{E} \mid f_{1}(h)(X, Y)-$ $f_{2}(h)(X, Y) \mid$. Condition 1 implies that $\mathbf{E}\|h(X, Y)\|<\infty$. Given $\varepsilon>0$ there exists a simple (measurable, finitely valued) symmetric kernel $\widetilde{h}$ such that $\mathbf{E}\|h(X, Y)-\widetilde{h}(X, Y)\|<\varepsilon$. It follows that $\left\{f(\widetilde{h}(X, Y)): f \in B^{*},\|f\| \leqslant 1\right\}$ is totally bounded in $L_{1}$ and therefore $\left\{f(h(X, Y)): f \in B^{*},\|f\| \leqslant 1\right\}$ is totally bounded in $L_{1}$.

We will check condition (2): for each $f_{1}, \ldots, f_{k} \in T$, the vector $\left(V_{n}\left(f_{1}(h)\right), \ldots\right.$, $\left.V_{n}\left(f_{k}(h)\right)\right)$ satisfies the LDP with speed $1 / n$ and a good rate function. Notice that for every $k \in \mathbf{N}$ the product space $\left(l_{2}\right)^{k}$ is a real separable Banach space. Consider for each $f_{1} \circ h, \ldots, f_{k} \circ h$ the same expansion as in Section 2, that is, $\pi_{2} f_{l} \circ h(x, y)=$ $\sum_{r=1}^{\infty} c_{r, f_{l}} \psi_{r, f_{l}}(x) \psi_{r, f_{l}}(y), c_{0, f_{l}}=1, \psi_{0, f_{l}}=2 \pi_{1} f_{l} \circ h-\mathbf{E} f_{l} h(X, Y)$, where we skip the dependence of $h$ in the notation. With

$$
X^{k}(n):=\left(\left(\left|c_{r, f_{l}}\right|^{1 / 2} \frac{1}{n} \sum_{i=1}^{n} \psi_{r, f_{l}}\left(X_{i}\right)\right)_{r=0}^{\infty}\right)_{l=1}^{k} \in\left(l_{2}\right)^{k}
$$

we obtain that the sequence $\left\{X^{k}(n)\right\}_{n \in \mathbf{N}}$ satisfies the LDP, if Conditions 1 and 2 are fulfilled for every $f_{l} \circ h, l=1, \ldots, k$. Therefore notice that every continuous, linear map on $\left(l_{2}\right)^{k}$ is described by an element $z=\left(z_{1}, \ldots, z_{k}\right) \in\left(l_{2}\right)^{k}$ via $f\left(x_{1}, \ldots, x_{k}\right)=\sum_{\nu=1}^{k}\left(\sum_{l=1}^{\infty} x_{\nu l} z_{\nu l}\right)$ for every $x=\left(x_{1}, \ldots, x_{k}\right) \in\left(l_{2}\right)^{k}$ with the notation $x_{\nu}=\left(x_{\nu r}\right)_{r=0}^{\infty} \in l_{2}$. Applying the result of Arcones we obtain the LDP for the sequence $\left\{X^{k}(n)\right\}_{n \in \mathbf{N}}$ if Conditions 1 and 2 are fulfilled for $f$ in the dual of $\left(l_{2}\right)^{k}$. Using the representation of each such $f$, both conditions follow by applying Hölder's inequality and Conditions 1 and 2, given for each $f_{l} \circ h, l=1, \ldots, k$.

Let us define the continuous function $F_{h}^{k}:\left(l_{2}\right)^{k} \rightarrow \mathbf{R}^{k}$ by

$$
F_{h}^{k}\left(y_{1}, \ldots, y_{k}\right):=\left(y_{l 0}+\sum_{r=1}^{\infty} \operatorname{sign}\left(c_{r, f_{l}}\right) y_{l r}^{2}\right)_{l=1}^{k}
$$

with the notation $y_{l}=\left(y_{l r}\right)_{r=0}^{\infty}$, we obtain the representation $\left(V_{n}\left(f_{1}(h)\right), \ldots, V_{n}\left(f_{k}(h)\right)\right)=$ $F_{h}^{k}\left(X^{k}(n)\right)$. Using the contraction principle, the LDP for the vector follows. We obtain that the vector $\left(U_{n}\left(f_{1}(h)\right), \ldots, U_{n}\left(f_{k}(h)\right)\right)$ satisfies the LDP with the same rate function: for $F_{k}:=\left(f_{1} \circ h, \ldots, f_{k} \circ h\right)$ we get

$$
\int_{S^{2}} \exp \left(\lambda\left\|F_{k}(x, x)\right\|\right) d \mu^{\otimes 2}<\infty
$$

for some $\lambda>0$, choosing the norm $\|\cdot\|$ on $\mathbf{R}^{k}$ defined by $\left\|\left(x_{1}, \ldots, x_{k}\right)\right\|=\left|x_{1}\right|+\cdots+\left|x_{k}\right|$ and applying Hölder's inequality and condition (1.1) for every $f_{l} \circ h$. With the proof of Theorem (1.10) in [6] we obtain the LDP for $\left(U_{n}\left(f_{1}(h)\right), \ldots, U_{n}\left(f_{k}(h)\right)\right)$ as in the proof of Theorem 2.2.

In the last step we will check that

$$
\lim _{\eta \rightarrow 0} \limsup _{n \rightarrow \infty} \frac{1}{n} \ln \mathbf{P}\left\{\sup _{d\left(f_{1}, f_{2}\right) \leqslant \eta}\left|U_{n}\left(f_{1}(h)\right)-U_{n}\left(f_{2}(h)\right)\right| \geqslant \tau\right\}=-\infty
$$

for each $\tau>0$. This is a bit involved.

Set $h_{\eta}:=\sup _{d\left(f_{1}, f_{2}\right) \leqslant \eta}\left|f_{1}(h)-f_{2}(h)\right|$. Note that

$$
\sup _{d\left(f_{1}, f_{2}\right) \leqslant \eta}\left|U_{n}\left(f_{1}(h)\right)-U_{n}\left(f_{2}(h)\right)\right| \leqslant U_{n}\left(h_{\eta}\right) .
$$

Hence by assumption (3.1), for any $\lambda>0, \Psi_{h_{\eta}}(\lambda):=\mathbf{E} \exp \left(\lambda h_{\eta}\left(X_{1}, X_{2}\right)\right)=$ $\mathbf{E} \exp \left(\lambda Y^{\eta}\right)<\infty$, for all $\eta>0$ sufficiently small. Moreover, with $k:=\lfloor n / 2\rfloor$, we obtain that

$$
\mathbf{E} \exp \left(\lambda k U_{n}\left(h_{\eta}\right)\right) \leqslant \Psi_{h_{\eta}}^{k}(\lambda)
$$

(Hoeffling's inequality, see, for example, [10, Lemma C, page 200]). 
Further, since $h_{\eta}\left(X_{1}, X_{2}\right) \stackrel{\mathbf{P}}{\longrightarrow} 0$ as $\eta \rightarrow 0$, for any choice of $\tau>0$, we have for all $\eta>0$ sufficiently small both

$$
\theta(\eta):=\mathbf{E}\left(h_{\eta}\left(X_{1}, X_{2}\right)\right)<\frac{\tau}{2}
$$

and

$$
\begin{aligned}
\Psi_{h_{\eta}}(\lambda) e^{-\lambda \theta(\eta)} & =\mathbf{E} \exp \left\{\lambda\left(h_{\eta}\left(X_{1}, X_{2}\right)-\theta(\eta)\right)\right\} \\
& \leqslant 1+\frac{\lambda^{2}}{2} \mathbf{E}\left(\left(h_{\eta}\left(X_{1}, X_{2}\right)-\theta(\eta)\right)^{2} \exp \left(\lambda\left|h_{\eta}\left(X_{1}, X_{2}\right)-\theta(\eta)\right|\right)\right) \leqslant 1+\frac{\lambda \tau}{4} .
\end{aligned}
$$

Thus

$$
\begin{aligned}
\mathbf{P}\left\{U_{n}\left(h_{\eta}\right) \geqslant \tau\right\} & \leqslant \mathbf{P}\left\{U_{n}\left(h_{\eta}\right)-\theta(\eta) \geqslant \frac{\tau}{2}\right\} \leqslant e^{-k \lambda \tau / 2} \mathbf{E} \exp \left(k \lambda\left[U_{n}\left(h_{\eta}\right)-\theta(\eta)\right]\right) \\
& \leqslant e^{-k \lambda \tau / 2}\left(\Psi_{h_{\eta}}(\lambda) e^{-\lambda \theta(\eta)}\right)^{k} \leqslant e^{-k \lambda \tau / 2}\left(1+\frac{\lambda \tau}{4}\right)^{k} \leqslant e^{-k \lambda \tau / 4}
\end{aligned}
$$

From this bound we immediately get that for each choice of $\tau>0$ and any $\lambda>0$

$$
\lim _{\eta \rightarrow 0} \limsup _{n \rightarrow \infty} \frac{1}{n} \ln \mathbf{P}\left\{U_{n}\left(h_{\eta}\right)>\tau\right\} \leqslant-\frac{\lambda \tau}{8}
$$

which by the arbitrary choice of $\lambda$ and the inequality in (3.3) implies (3.2).

$\mathrm{R}$ e $\mathrm{m}$ a r k 3.1. Theorem 3.1 holds for any degree $m \geqslant 2$. This follows using the spectral decomposition introduced in Section 2 as well as the facts that (2.5) is proved for arbitrary degree in [6] and that the decomposition arguments using Baranyai's result are applicable for any degree $m \geqslant 2$, see [6].

Acknowledgment. I would like to thank Prof. D. Mason for fruitful discussions concerning an easier proof of (3.2).

\section{REFERENCES}

1. Arcones M. Large deviations of empirical processes. - High Dimensional Probability III. Ed. by J. Hoffmann-Jørgensen, M. B. Marcus, and J. A. Wellner. Boston: Birkhäuser, 2003, p. 205-223.

2. Arcones M. A. Large deviations for $U$-statistics. - J. Multivariate Anal., 1992, v. 42, № 2, p. 299-301.

3. Dembo A., Zeitouni O. Large Deviations Techniques and Applications. New York: Springer-Verlag, 1998, 386 p.

4. Donsker M., Varadhan S. Asymptotic evaluation of certain Markov process expectations for large time. III. - Comm. Pure Appl. Math., 1976, v. 29, p. 389-461.

5. Eichelsbacher $P$. Large deviations for products of empirical probability measures in the $\tau$-topology. - J. Theoret. Probab., 1997, v. 10, № 4, p. 903-920.

6. Eichelsbacher P., Schmock U. Large deviations of $U$-empirical measures in strong topologies and applications. - Ann. Inst. H. Poincaré, Probab. Statist., 2002, v. 38, № 5, p. 779-797.

7. Hoeffding $W$. Probability inequalities for sums of bounded random variables. - J. Amer. Statist. Assoc., 1963, v. 58, p. 13-30.

8. Lee A.U-Statistics: Theory and Practice. New York: Dekker, 1990, 302 p.

9. Schied A. Cramer's condition and Sanov's theorem. - Statist. Probab. Lett., 1998, v. 39 , № 1 , p. 55-60.

10. Serfling R. J. Approximation Theorems of Mathematical Statistics. New York: Wiley, $1980,371 \mathrm{p}$.

11. Sethuraman $J$. On the probability of large deviations of families of sample means. Ann. Math. Statist., 1964, v. 35, p. 1304-1316. 